\title{
STATIN THERAPY FOR PRIMARY PREVENTION
}

Statin therapy has now been shown to reduce the incidence of adverse cardiovascular events and mortality in individuals without overt signs of cardiovascular disease but with risk factors, which indicates that this population should be considered for prophylactic therapy.

Statins are known to be effective in reducing risks of adverse outcomes in patients with established cardiovascular disease-that is, as a secondary preventive measure. However, it is not known whether statin therapy would be useful in preventing the onset of adverse events in individuals without established cardiovascular disease and, therefore, regarded as being at low risk. To assess the effectiveness of statin therapy as a primary preventive measure, researchers in The Netherlands, Japan and the US conducted a meta-analysis of randomized trials that focused on primary prevention. Their study also aimed to clarify whether the effects of statins are influenced by patients' age or sex, or the presence of diabetes, as it is not clear from previous studies whether statin therapy benefits women, patients over the age of 65 years, or patients with diabetes.

A search of the Cochrane Central Register of Controlled Trials identified 10 randomized trials - representing a total of 70,388 participants-that met the following inclusion criteria: trial participants had been randomly assigned to receive statin treatment or to a control group; participants had been followed up for at least 1 year; the studies were focused on primary prevention (at least $80 \%$ of the participants had no history of cardiovascular disease); and data on the incidence of cardiovascular events and mortality were available. A subgroup analysis of data from the ALLHAT study and five other trials was also conducted to assess the influence of patients' age and sex, and the presence of diabetes on the effectiveness of statin treatment.

Participants with an average age of 63 years had been randomly assigned to either a statin-treated group $(n=35,138)$ or a control group $(n=35,250)$, and were followed up for an average of 4 years. Approximately one-third of the participants were women and onequarter had diabetes, which represented a larger proportion of these subgroups than in previous statin trials. Statin therapy significantly reduced all-cause mortality by $12 \%$, major adverse coronary events by $30 \%$ and cerebrovascular events by $19 \%$. Comparable effects of the treatment were observed across the subgroups, indicating that the effectiveness of statin therapy was not influenced by patients' age or sex, or by the presence of diabetes. These findings indicate that statin therapy would be useful as a primary preventive measure to reduce the risk of adverse cardiovascular outcomes in individuals considered to be at low risk. "Our data suggest that people without established cardiovascular disease should not be denied the relative benefits of long-term statin use," says Jasper Brugts of the Erasmus Medical Center in The Netherlands. "The exact threshold for cost-effectiveness of such treatment should be investigated further and will probably depend on the level of risk due to specific combinations of risk factors for cardiovascular disease," he adds.

Sharmini Rajanayagam

Original article Brugts, J. J. et al. The benefits of statins in people without established cardiovascular disease but with cardiovascular risk factors: meta-analysis of randomised controlled trials. BMJ 338 , b2376 (2009). 